\title{
Phytodiversity of Raiganj Wildlife Sanctuary (Kulik Bird Sancturay) of Uttar Dinajpur District in West Bengal, India
}

\author{
Debabrata Das ${ }^{1}$ and Pampi Ghosh ${ }^{2}$ \\ 1- Microbiology and Ecology Laboratory, Post Graduate Department of Botany, Darjeeling Govt. College, \\ Darjeeling, West Bengal, India. \\ (Ex-Asst. Prof. of Botany, Jhargram Raj College, Jhargram, Paschim Medinipur, West Bengal and Ex- \\ JRF/SRF-ICFRE Project at CNH, Gope Palace, EX-JRF/SRF-Flora India Project, Central National \\ Herbarium, Botanical Survey of India, Shibpore, Howrah, West Bengal, India) \\ 2-Department of Botany, Seva Bharati Mahavidyalaya, Kapgari, Paschim Medinipur, West Bengal, India. \\ (Ex-Headmistress, Pasang Girls' High School, Pasang, Debra, Paschim Medinipur, Ex-Assistant Teacher of \\ Baidara Janakalyan High School, Uttar Dinajpur, West Bengal, and Jahangirpur High School, Dakshin \\ Dinajpur, West Bengal)
}

\begin{abstract}
The present paper represents the ecosystem along with the Phytodiversity of angiospermic plants occurring in and around Raiganj Wildlife Sanctuary (Kulik bird Sanctuary) with potential ecological significance at Raigang, Uttar Dinajpur, West Bengal. The area of the place revealed 224 species of angiosperms through extensive survey since 1999 to 2014 with full potential of screening and examinations. It also includes Cycadaceae and Pteridophytes in different aspects. This type of work may be regarded as the pioneer work for further more elaborate studies of Angiosperms for documentation or studies in different aspects in the field of Biology. This means that, it has its ecological meaning and environmental significance, because complete works regarding diversity of plants have not yet been published for crucial analysis previously.
\end{abstract}

Keywords: Kulik-Uttar Dinajpr, Species of Plants and Birds, Cronquist's Classification, Nature and Natural Resources.

\section{Introduction}

Raiganj Wildlife Sanctuary is popularly called Kulik Bird Sanctuary in West Bengal. Kulik forest is a forest under Social forestry of this area mainly dominated by Pituli, Jarul, Hijal, Chhatim and Kalkasunda along with flagship species like Bat and Pakur. Kulik forest, situated on National Highway 34, is only $2 \mathrm{~km}$ away from the Raiganj Central Bus Stand. It was established and working mainly for the conservation of birds of migratory kind. The government of West Bengal gave it as a full form of Sanctuary on the $11^{\text {th }}$ day of April 1985 under Govt. Order (G.O/No. 1901-FOR 86/82). The sanctuary is situated in Bhattardighi, Abdulghata and on Sohari Mouzas of Raiganj block, Uttar Dinajpur and near the side of National Highway No. 34. River Kulik bound it at the northeast to partially the southeast. The sanctuary is situated in between $25^{0} 37^{\prime} \mathrm{N}$ latitudes to $88^{\circ} 12^{\prime} \mathrm{E}$ longitudes. Chanditala and Kamarpara villages in the east of Sanctuary Kulik, on the west it is bounded by Poliapara, in south by Munipara and Manirpur in the north. The sanctuary is spread over an area 1.30 square kilometer in which 1.16 square kilometer is buffer area (286.23 acre) and 0.14 square kilometer ( 35 acre) is core area (Anonymous). It is situated under Karnajora Range of Raiganj Social Forestry Division. The site is diverse because, so many wild medicinal plants as well as shrubby plants of different types available with high density even other types like bryophytes, pteridophytes, fungi, algae and nematodes of different types boost there with typical association. A large no of tree species found here along with some exotics like Eucalyptus and Cassias available with high degree of consociations. Rare plants of potential conservational values also found which share the dignity of this paradise for exotic birds of migratory kind. It also provided with enormous water bodies filled up by the water of Kulik River in which core area is located at the centre surrounded by water. Common predators available here are wildcat, Jackal, Lizard, Bat, Spider, Serpentine snake, mouse, rat, squirrel, mole etc. The yellow monitor lizard, the rare species of reptiles are also found here and there. The main attraction of this site is for the birds. The native have their permanent address in this sanctuary are the bulbul, dove, sparrow, crow, vulture, eagle, parakeet, owl, woodpecker, kingfisher, duck, drongo and cuckoo. The migratory birds like night heron, cormorant, little cormorant, egrets, and open-billed storks visit since end of May to $1^{\text {st }}$ week of July in each year from South Asia and from Coastal Points. According to Basu and Sah (2013), the open- Billed storck comes in the last week of June at the Kulik Bird Sanctuary stays there for 5-6 months and leaves the place in the month of December of every year. According to the report of forest guard of the same range, the departure 
time of migratory birds begin in the mid December and extended up to the end of the January each year. With in the short period of time they lay eggs, which is July to August in each year. They choose bushy trees for nesting. Species involved for nesting at Kulik found are Ficus benghalensis (Bat), Anthocephallus cadamba (Kadamba), Alstonia scholaris (Chhatim), Barringtonia acutangula (Hijal), Ficus glomerata (Dumur), Terminalia arjuna (Arjun), Dalbergia sissoo (Sissoo), Lagerstroeimia speciosa (Jarul), Streblus asper (Seora), Trewia nodiflora (Pituli), etc. (Basu and Sah, 2013). The materials they need for nesting collected from nearby environment. The nest building materials contained of fragments of old branches of trees, some soft green leaves and grasses. These branches helped in strengthening and the leaves and grasses helped in softening of the nest (Pramanik et al., 2009). A large number of twigs with green leaves of Eucalyptus they collect for the purpose of nesting are due to their medicinal or anti-germicidal values. The mean temperature of this site is 26 degree Celsius. Rainfall is more or less $1100 \mathrm{~mm}$ whereas the relative humidity is $89 \%$.

The interactions between plants and birds are unique because they need food, nesting materials and shelter which comes from plants of the sanctuary and nearby. So, uniqueness is diversity and pattern is mosaic which are interacting cyclic phases year after year for the liveliness and their protection in and around nature. In addition to local birds other birds available here are migratory one.

The open- Billed storks comes in the last week of June at the Kulik Bird Sanctuary stays there for 5-6 months and leave the place in the month of December of every year. The similar pattern is observed in Ghoragata of Howrah District where three trees are the temporal habitats for the same bird species. Other places are Bagnan, Santragachi, Sankrail which found in the same district for temporal stay of birds including the open billed stork. In Kendua village of Jamboni block in Jhargram of Paschim Medinipurn district the same pattern of temporal habitats of migratory birds have been observed. Tentul trees (Tamarindus indica) and Khiris trees (Samanea saman) are the habitats of the birds at Jamboni area. The birds choose special types of plants for nesting. Here, Species involved for nesting found are Ficus benghalensis (Bat), Anthocephallus cadamba (Kadamba), Alstonia scholaris (Chhatim), Barringtonia acutangula (Hijal), Ficus glomerata (Dumur), Terminalia arjuna (Arjun), Dalbergia sissoo (Sissoo), Lagerstroeimia speciosa (Jarul), Streblus asper (Seora), Trewia nodiflora (Pituli), etc. (Basu and Sah, 2013). The habitat for the specific purpose they opt for the protection as well as for health conjugal environment. In connection of the above kinds other parameters they used for the association in which they feel association. The site is diverse with medicinal Plants which boosts nature made conservatory. The naturally occurring plants along with plantation stands are found in the social forestry site with admixed Eucalyptus, Trewia (Pitali) along with Lagerstroemia plant (Ghosh, 2004).

Vegetation is a composition of plant species. So, before going to study the vegetation, taxonomy and species inventory study is essential for any site. Remembering the theme, Dennis and Ruggiero (1996) suggested four possible approaches for orienting an inventory namely-(1) Survey of major elements; (2) Identification of key stone species and indicator elements; (3) Identification of target elements, such as threatened species, and (4) Comprehensive assessment of all other important elements, such as exotic or alien species/invasive species, flagship species, and economically useful taxa. The purpose and orientation of an inventory is always for the raise of data to enrich data bank for proper management of ecosystem and some of the good data over the old one are called indicator data which helps to study the quality health of an ecosystem. The inventory, therefore will determine the choice of material and methods, which on other hand will influence the completeness of the inventory in terms of taxonomy, community, ecosystem dynamics, strategies of development, fluctuation of elements over time and space, geographical space, seasonal variations of the occurrence, temporal representation, stability and functioning etc. (Das, 2014; Solbrig, 1991; Stohlgren and Quin, 1991). Remembering the theme in mind the present study has been taken for consideration to fulfil the first two objectives as a whole. Therefore, the present phytodiversity studies in connection with the ecosystem have been taken.

\section{Study Area}

The study area is Kulik at Raiganj of Uttar Dinajpur District. The district is surrounfded by Dakshin Dinajpur in the southeast, by Bangladesh in the East, by Malda in the south, by Kishanganj of Bihar in the west and Purnia in the southwest part. The community development block in which it is situated is Raiganj. The nearest Railway station is Raiganj and the headquarter is situated at Karnajora of West Bengal (Map 1,2). Study area is social forestry site including some villages nearby which having mesophytic vegetation along with some agricultural varieties and horticultural species. River Kulik partially surrounding the area with some water bodies which boost luxuriant growth of various weeds as well as some aquatic plants of economic importance. 


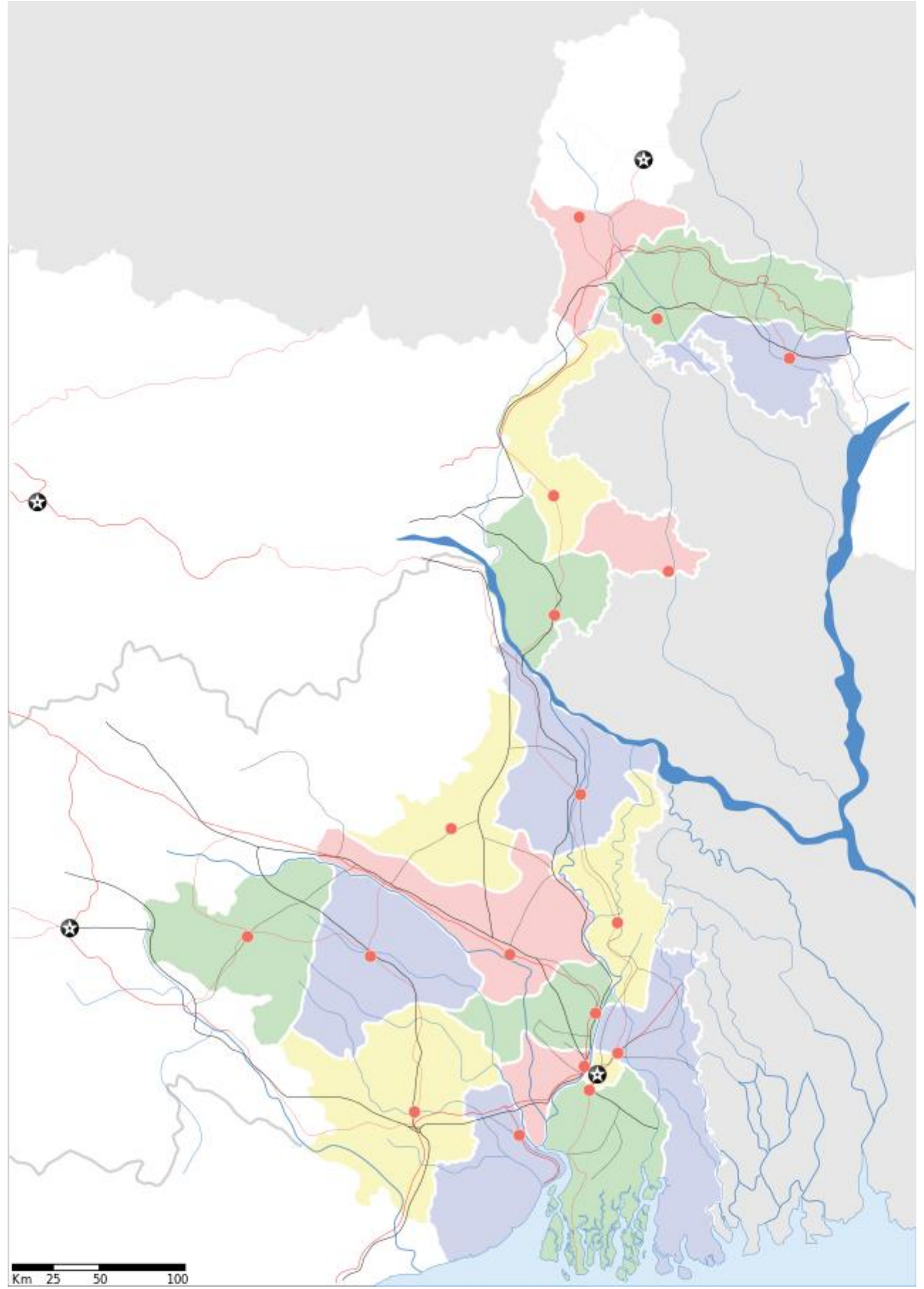

Map 1. Map of West Bengal, Courtesy: Wikipedia 


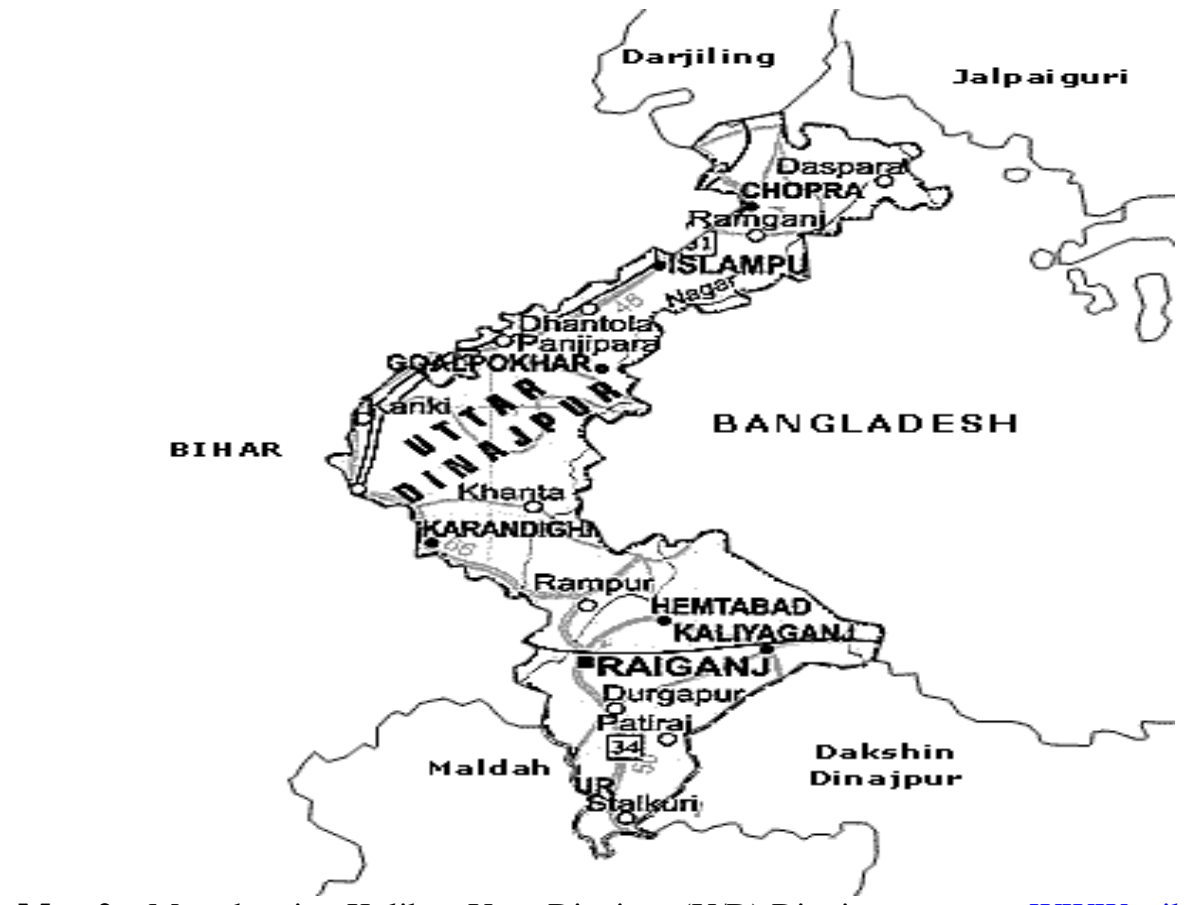

Map 2. Map showing Kulik at Uttar Dinajpur (U/D) District, courtesy: WWW.wikipedia

Climate

The area is mixed humid type with alluvial soil best for cultivation. Soil is fertile due to older alluvium deposited which is falls under the basin of Rajmahal hills on the east. The main composition is rainfall and humidity with variable temperature. The altitude is almost about $30 \mathrm{mt}$. above mean sea level (MSL). Temperature maximum 25 degree and minimum 21 degree centigrade. During winter the maximum and minimum temperature is 23 and 9 degree centigrade respectively. Rainfall is about $155 \mathrm{~cm}$ (July to Sep.) round a year (wiki information).

\section{Vegetation}

Here vegetation is mixed deciduous type with some plantation sites. Species of economic types are Cocos nucifera, Artocarpus heterophyllus, Olea sp., Dalbergia sissoo, Gmelina arborea, Anthocephalus cadamba, Terminalia chebula, Albizia lebbeck, Samanea saman, Flagship species found here are Ficus benghalensis, F. hispida, F. glomerata, F. cunea etc. Weeds of exotic types in aquatic bodies are species like Tillanthera and Eichhornia sp. along with Salvinia and Azolla sp., whereas in the bundh or semi-aquatic bodies the most promising species are Veteveria zizanoides and Impereta cylindrica. Weeds of fallow land are Jussia sp., Ludwigia sp., Mimosa sp., Eupatorium sp., Alysicarpus sp., Evolvulus sp., Tephrosia sp., Sonchus sp., Spilanthes sp., Achyranthes sp., Spermacoce sp., Oldenlandia sp., Phylla sp., etc. In waste land the species like Hibiscus vitifolius, Leonotis nepetifolia, Anisomeles indica, Hyptis suaveolens, Daemia extensa, Sida acuta, Sida cordata, Melochia corchorifolia, Clerodendrum sp., Parthenium are common along with Solanum sp. Other species found here are Cuscuta sp., Dendropthoae sp., as plant parasite. Some orchids of epiphytic kind are also available here. Trees found here are Terminalia bellerica, T. chebula, T. catappa, Bombax ceiba, Holarrhena antidysenterica $(=H$. pubescens), Cassia fistula, Lagerstroimeia indica, Aegle marmelos, Semecarpus anacardium, Butea frondosa etc. Woody climbers found here are Tiliocora racemosa, Hemidesmus indicus, Ichnocarpus pubescens, Bauhinia vahlii, Spatholobus roxburghii, Aganosma dichotoma, Cephalendra indica, Trichosanthes pametta, Jasminum dispermum, J. sambac, Dalbergia stipulacea. Species producing wood of commerce are Samania saman, Albizzia lebbeck. Other species found are Cereus hexagonus, Opuntia sp., which found in dry and open land habitats. Aristolochia indica, Curculigo orchioides, Diospyros tomentosa, D. sylvatica, Clerodendrum indicum etc. are medicinal plants. Dillenia indica, Mangifera indica, Ficus religiosa, F. benghalensis, F. hispida, Gelonium multiflorum, Glochidion lanceolarium, Annona squamosa, A. reticulate, Zizyphus rugosa, Flacourtia ramaontchii, Pterospermum acerifolium, Tectona grandis, Borassus flabellifer, Phoenix sylvestris, Cassia ocidentalis, Calotropis procera, C. gigantean, Leonotis nepetifolia, Martynia annua, Tribulus terrestris, etc are common available here and there. Plantation sites include species like Eucalyptus, Acacia, Anacardium, Simarouba, Disoxylum, Anthocephalus, Phyllanthus, Couripita guanensis, Mesua sp. etc. along with a lot of Bamboos. Ornamentals available here are Caesalpinia sp., 
Delonix sp., Alamanda sp., Thunbergia sp., Ixora sp., Gardenia sp., Musa sp., Ravenala sp., Heliconia sp., Cammelia sp., Hibiscus sp., Salvia sp., Croton sp, Jasminum sp., Polyanthes sp., Rosa sp., Tagetes sp., Dahlia sp., Chrysanthemum sp. etc.

\section{People}

Main composition of the human resource in the said area is filled by backward classes. The area comprises people who speak Bengali, Urdu, Hindi and Maithili languages. The specific backward communities are more but a common group available here are Polia and Rajbanshi.

\section{Cultivars}

The soil is alluvial type due to great deposition of old alluvium from Rajmahal hills. Therefore, cultivated crops of dominant types are Rice, Jute, Mesta and Sugarcane. Other underground tuberous and corm like cultivars are Alocasia, Colocasia, Zinger and Amada. Nearby sericulture range have been found which producing Morus plants.

\section{Medicinal Plants}

The area has good potential of medicinal plants. These are species like Cassia fistula, Cissus quadrangularis, Pongamia pinnata, Achyranthes aspera, Acorus calamus, Allium sativum, Andrographis paniculata, Vitex negundo, Tinospora cordifolia, Withania somnifera, Rauvolfia serpentina, Rauvolfia tetraphylla, Morinda citrifolia, Mimosa pudica, Kalanchoe pinnata, Hemidesmus indicus, Tylophora asthmatica, Gymnema sylvestre, Euphorbia nerifolia, Datura metel, Ocimum sanctum, Ocimum gratisimum, Calotropis procera, C. gigantean, Azadirachta indica, Solanum indicum, Amorphophalus bulbifera, Streblus asper, Alstonia scholaris, Aristolochia indica, Stephania hernandifolia, Cissus adnata, Curculigo orchioides, Polygala crotalarioides, Zizyphus jujuba, Z. rugosa, Zornia diphylla, Adhatoda vasica, Mollugo pentaphylla, Enhydra fluctuens, Wedelia calandulacea, Vernonia cinerera, Solanum trilobatum, Solanum nigrum, Solanum xanthocarpum, Ipomoea carnea, I. aquatic, Glenus lotoides, Moringa oleifera, Morinda citrifolia, Agave Americana, Spondias dulcis, Scoparia dulcis, Averhoeea carambola, Meyna spinosa etc.

\section{Exotic floral Species}

The most promising exotic species found here are Lantana camara, Chromolaena odorata, Hyptis suaveolens, Parthenium hysterophorus, etc. which is naturalized in the said area unlikely covers most of the areas and cause threat to local flora.

Fauna

As the forest is under social forestry so, numbers of local kinds of wild animals are available here less in number, maximum are residential from other side as they posses temporal stay. The good example is Storks and Cormorants. So, other faunal members are Porcupine, Snakes, Lizards, Butterflies, Insects, Ants, Spider, monitor and common birds. Birds available here are Sparrow, kingfisher, Crane, Crow, Raven, Dove, Parrot and Parakeet, Mayna, white breasted water hen etc. Pigeon and Drongos are available here in the area where cultivated crops are common.

\section{Materials and Methods}

Study was made in three seasons i.e. summer, monsoon and in winter round the year for analysis of vegetation since 1999 till date including phyto-diversity study. In this particular region three seasons namely monsoon (July-October), winter (November-February) and summer (March-June) are well perceived. During study some plant specimens were collected and Herbarium specimens were prepared as per the methods of Rao and Sharma, 1990. The specimens were collected and processed for presentation as herbarium specimens and for identification using botanical and ecological standard. Specimens were carefully studied, critically examined and cross checked with the specimens housed in the CAL herb, BSI, Shibpore, Howrah. For conformity of specimens, local floras were consulted (Prain, Vol-I-II, 1903; Hains, Vol-I-III, 1921, Hooker, 1892-1897). To consult some publications, Taxonomy and similar research papers from website have been downloaded and followed by Ghosh, 2014; Das and Das, 2014; Ghosh and Das, 2014, Das, 2014b. Some books published by West Bengal Forest Directorate, Research Wing (Anonymous 2005, 2010), BSI, Kolkata (Anonymous 1997) have also been consulted to analyze the report along with our collections that the plants are either medicinal or not. Methodology used for abundance study followed by Groom et al., (2006) along with the thesis of Das (2007). Relevant literature have been collected and consulted for the preparation of the manuscript. The voucher specimens were housed in our custody. For birds study paper made by Pramanik et al. (2009), Sharma (2007) 
have been consulted which gave us a comprehensive idea about the ecosystem diversity of birds in connection with the floral elements.

\section{Results}

Systematic Enumeration of Taxa made as per Cronquist (1988):

Magnoloiopsida: (Dicotyledons) :

Sub Class: I Magnoliidae

Family: 8. Annonaceae

Species: Annona reticulate L., A. Squamosa L., Artabotrys hexapetalus (L. F.) Bhandari; Polyalthia longifolia Benth. \& Hook.f.; Polyalthia longifolia var. Pendula (Thw.) Sonn.

Polyalthia simiarum Benth. \& Hook.f.

Order: 3. Piperales

Family: Piperaceae

Species: Piper longum L.; Piperomia pellucida (L.) HB\&K.

Order: 4. Aristolochiales

Family: Aristolochiaceae

Species: Aristolochia indica L.

Order: 6. Nympheales

Family: Nymphaeaceae

Species: Nymphaea rubra Roxb. ex Salisb.;

Family: Ceratophyllacae

Species: Ceratophyllum demursum L.

Order: 7. Ranunculales

Family: Ranunculaceae

Species: Clematis gouriana Roxb. ex DC.; Ranunculus sclerus L.

Order: 8. Papaverales

Family: Papaveraceae

Species: Argemone maxicana L.;

Sub-Class: II Hamamelidae

Order: 6. Urticales

Family: Ulmaceae

Species:Holoptelea integrifolia (Roxb.) Planch.; Trema orientalis (L.) Bl.

Family: Cannabinaceae

Species: Cannabis sativa L.

Family:Moraceae

Species: Artocarpus integrifolia L. f.; Artocarpus lakoocha Roxb.; Ficus benghalensis L.; Ficus cunea Ham.; Ficus elastic L.; Ficus hispida L.f.; Ficus religiosa L.; Streblus asper Lour.

Family: Urticaceae

Species: Laportea interupta (L.) Chew; Pilea microphylla (L.) Liebm.; Pouzolzia zeylanica (L.) Benn.

Order: 11. Casuarinales

Family: Casuarinaceae

Species: Casuarina equisetifolia J.R. \& G. Forst.

Sub-Class III Caryophyllidae

Order: 1. Caryophyllales

Family: Nyctaginaceae

Species: Boerhaavia diffusa L.; 
Family: Cactaceae

Species: Opuntia vulgaris Mill.

Family: Amaranthaceae

Species: Achyranthes aspeera L.;

Family: Molluginaceae

Species: Mollugo pentaphylla $L$.

Order: 2. Polygonales

Family: Polygonaceae

Species: Polygonum hydropiper L.;

Order: 2. Plumbaginales

Family: Plumbaginaceae

Species:Plumbago zeylanica $\mathbf{L}$.

Order: 3. Malvales

Family:Tiliaceae

Species:Corchorus aestuens L.; Triumfetta rhomboidea Jacq.

Family: Sterculiaceae

Species: Abroma augusta L.; Buettneria herbacea Roxb.; Melochia corchorifolia L.

Family: Bombacaceae

Species: Bombax ceiba L.; Ceiba pentandra (L.) Gaertn.

Family: Malvaceae

Species: Abutilon indicum Sweet; Hibiscus ros-sinensis L.; Hibiscus vitifolius L.; Malachra capitata L.; Sida acuta Burm.f.; Sida cordata Bross.; Sida cordifolia L. Urena lobata L.

Order: 4. Lecythidales

Family:Lecythidaceae

Species:Barringtonia acutangula (L.) Gaertn.;

Order: 6. Violales

Family:Flacourtiaceae

Species:Flacourtia indica Merr.

Family: Cucurbitaceae

Species: Coccinia grandis Voigt.; Trichosanthes cucumerina $\mathbf{L}$.

Order: 8. Capparales

Family:Capparacaea

Species:Capparis sepiaria L.; Cleome gynandra L.; Cleome viscose L.; Crateva nurvula Buch.-Ham.

Family: Brassicaceae

Species: Rorippa indica Hiren.;

Family: Moringiaceae

Species: Moringa oleifera Lamk.

Order: 12. Ebenales

Family:Sapotaceae

Species:Mimusops elengi L.;

Family: Ebenaceae

Species: Diospyros sylvatica Roxb.; Diospyros montana Clke., D. embryopteris Pers., D.paniculata Dalz.

Sub-Class V. Rosoidae

Order: 1. Rosales

Family:Crassulaceae

Species:Bryophyllum calycinum Salisb.

Order: 2. Fabales

Family:Mimosaceae 
Species:Acacia auriculoformis A. Cunn.; Acacia nilotica L.,; Acacia farnesiana (L.) Willd.; Albizia lebbeck Willd.; Leucaena glauca Benth.; Mimosa pudica L.; Pithecellobium dulce Benth.; Samanea saman Merr.

Family: Caesalpiniaceae

Species: Bauhinia variegate L.; Cassia alata L.; Cassia fistula L.; Cassia occidentalis L.; Cassia siamea Lamk., Cassia sophera L.; Cassia tora L.; Delonix regia Raf.; Peltophorum pterocarpum Baker ex Heyne Family: Fabaceae

Species: Abrus precatorius L.; Alysicarpus vaginalis DC; Crotalaria pallid Aiton; Desmodium gangeticum DC.; Desmodium triflorum DC.; Glericidia sepium Kunth ex Steud; Zornia gibbos Span.

Order: 6. Myrtales

Family:Lythraceae

Species: Lagerstroemia speciosa Pers.; Lawsonia inermis L.

Family: Myrtaceae

Species: Callistemon linearis DC.; Eucalyptus maculate (Hook.) Baily; Psidium guajava L.; Syzygium cumuni Skeels

Family: Onagraceae

Species: Ludwigia adscendens Hara; Ludwigia perrenis L.

Family: Combretaceae

Species: Terminalia arjuna Wt. \& Arn.; Terminalia crenulata Roth

Order: 12. Euphorbiales

Family: Euphorbiaceae

Species: Acalypha indica L.; Chrozophora rottleri Juss.; Croton bonplandianum L.; Euphorbia hirta L.; Euphorbia prostate W. Ait.; Phyllanthus fraternus Webster; Phyllanthus reticulates Poir.; Ricinus communis L.; Trewia nudiflora $L$.

Order: 13. Rhamnales

Family: Rhamnaceae

Species: Ventilago denticulate Willd.; Zizyphus mauritiana Lamk., Zizyphus oenoplea Mill.

Family: Rhamnaceae

Species: Leea macrophylla Roxb.

Family: Vitaceae

Species: Ampelocissus latifolia Planch.; Cayratia trifolia Domin; Vitis quadrangularis Wall.

Order 16. Sapindales

Family: Sapindaceae

Species: Allophyllus serratus Radlk.; Cardiospermum helicacabum L.

Family: Anacardiaceae

Species: Mangifera indica L.; Lannea coromandelica Merr.

Family: Simaroubiaceae

Species: Ailanthus excels Roxb.;

Family: Meliaceae

Species: Azadirachta indica L.; Melia azedaracth L.; Swietenia macrophylla King.

Family: Rutaceae

Species: Citrus decumana L.; Muraya koenigii Spreng.

Family: Zygophyllaceae

Species: Tribulus terrestrois L.

Order: 18. Apiales 
Family: Apiaceae

Species: Centella asiatica Urban.

Sub-Class VI. Asteridae

Order: 1. Gentianales

Family: Loganiaceae

Species: Strychnos nux-vomica L.

Family: Gentianaceae

Species: Canscora diffusa R. Br. Ex Roem. \& Schult.

Family: Apocynaceae

Species: Alstonia scholaris R.Br.; Ichnocarpus frutescens R. Br.; Tabermontana divericata R. Br. Ex Roem. \& Schult.

Family: Asclepiadaceae

Species: Calotropis gigantean R. Br.; Cryptolepis buchanani Roem. \& Schult. ; Gymnema sylvestre R. Br. Ex Schult.; Hemidesmus indicus R. Br.; Tylophora indica Merr.

Order: 2. Solanales

Family: Solanaceae

Species:Nicotiana plumbaginifolia Viv.; Solanum indicum L.; Solanum nigrum L. ; Solanum sisymbrifolium Lamk.; Solanum surattense Burm. f.; Solanum torvum Swartz

Family: Convolvulaceae

Species: Evolvulus nummularius L., Ipomoea fistulosa Mart. Ex Cho.isy; Ipomoea obsura Ker-Gawl.

Family: Cuscutaceae

Species: Cuscuta reflexa Roxb.

Order: 3. Lamiales

Family: Boraginaceae

Species:Coldenia procumbens L.; Heliotropium indicum L.; Heliotropium strigosum Willd.;

Heliotropium ovalifolium Forsk.;

Family: verbenaceae

Species: Clerodendrum indicum O. Ktze.; Clerodendrum viscosum Vent.; Duranta repens L.; Gmelina arborea Roxb.; Phylla nodiflora Greene

Family: Lamiaceae

Species: Hyptis suaveolens Poit.; Leucas cephalotes Spreng.; Leucas lavendulifoliA Nees.; Ocimumgratisimum L.; Ocimum sanctum L.

Order:6.Scrophulariales

Family: Scrophulariaceae

Species: Lindernia antipoda Alst.; Lindernia ciliate Pen.; Lindernia opposetifolia Mukherjee; Mazus pumilus Steenis; Scoparia dulcis $\mathbf{L}$.

Family: Acanthaceae

Species: Adhatoda vasica Medic.; Andrographis paniculata Nees.; Dipteracanthus prostrates Nees; Hemigraphis hirta T.Anders; Justicia genderussa Burm. f.; Justicia simplex D. Don.; Rungia pectinata Nees.

Order: 8. Rubiales

Family: Rubiaceae

Species: Adina cordifolia Hook.f. ex Bran.; Anthocephalus cadamba Miq.; Dentella repens J.R.\& G.Forst.; Meyna spinosa Roxb.; Morinda tomrntosa Heyne ex Roth.; Oldenlandia corymbosa L.

Order:11. Asterales 
Family: Asteraceae

Species: Ageratum conyzoides L.; Blumea lacera Burm.; Chromolaena odorata King \& Robin.; Eclipta alba Hassk.; Elephantopus scaber L.; Emilia sonchifolia DC. Ex Wt.

Gnaphalium luteo-album L.; Launea asplanifolia Hook. f.; Spilathes achmella (Burm.f) DC.; Tridax procumbens L.; Vernonia cinerera Lees.; Xanthium strumarium L.

Class: Liliopsida (Monocotyledons)

Sub-Class II Arecidae

OrderI Arecales

Family: Aerecaceae

Species: Borassus flabellifer L.; Cocos nucifera L.; Phoenix sylvestris Roxb.

Order: 4. Arales

Family: Araceae

Species: Alocasia indica Schoot; Caladium bicolour Schoot; Colocasia esculenta Schoot; Typhonium trilobatum Schoot

Sub-Class: III. Commelinidae

Order 1. Commelinales

Family: Commelinaceae

Species: Commelina bengalensis L.; Cyanotis tuberose Schult.; Murdania nudiflora (L.) Brenan

Order 5. Cyperales

Family: Cyperaceae

Species: Cyperilus kyllinga Endl.; Cyperus rotundus L.; Scirpus articulates L

Family: Poaceae

Species: Bambusa nana Roxb.; Cynodon dactylon Pers.; Dendrocalamus strictus Nees; Desmostachya bipinnata Stapf.; Eragrostis sinoseurides..........; Oplismenus burmanii P.Beauv.; Paspalidum flavidum A. Camus; Vetiveria zizanoides Nash

Sub-Class: IV. Zingiberidae

Order: 2. Zingiberales

Family: Musaceae

Species: Musa paradisiacal L.; Ravenala madagascariensis Sonnerat

Family: Costaceae

Species: Costus speciosus J.E.Smith

Family:Cannaceae

Species: Canna indica $\mathbf{L}$.

Sub-Class: V. Liliidae

Order: Liliales

Family: Liliaceae

Species: Aloe vera Burm. f.; Asparagus racemosus Willd.

Family: Smilacaceae

Species: Smilax zeylanica $L$.

Family: Dioscoreaceae

Species: Dioscorea pentaphylla $L$.

Order: 2. Orchidacea

Family: Orchidaceae

Species: Vanda roxburghii R. Br.

Gymnosperms

Family: Cycadaceae 
Species: Cycas circinalis L.; Blota sp.; Microcycas sp.

\section{Pteridophytes}

Family: Ophioglossaceae

Species: Helminthostachys zeylanica Kaulf

Family: Polypodiaceae

Species: Lygodium flexosum Sw.; Pteris sp.;

Family: Adiantaceae

Species: Adiantum caudatum L.;

Family: Salviniaceae

Species: Salvinia natans Hoffim.

Family: Azollaceae

Species: Azolla imbricate R. Br.

Family: Marsileaceae

Species: Marsilia quadrifoliata $\mathbf{L}$.

\section{Discussion}

A statistical analysis of the plants reported from the sanctuary Kulik and its surroundings reveals that there are in total, 234 plant species of which 224 species under angiosperms whereas, 10 are under Gymnosperms and Pteridophytes. Out of total species studied, there are 78 families comprising of 64 dicots and 14 monocots (Table 1). There are a total number of 215 genera in which 184 belong to dicots and 31 to monocots. There are total 224 angiosperm species in which 192 dicots and 32 monocots. The ratio of monocot to dicot is 1:6. Among the families studied, dominant dicot families are Asteraceae (13 genera and 13 species), Euphorbiaceae (7 genera and 9 species), Mimosaceae (6 genera and 8 species). Similarly, monocot families of Poaceae (8 genera and 8 species), Araceae (4 genera and 4 species) are dominant one (Table 2). The species like Caladium, Ravenala, and Heliconia are ornamental elements in the Garden and found in front of the guesthouse of Sanctuary. The medicinal plants in the sanctuary available are Aristolochia indica, Boerhhavia diffusa, Achyranthes aspera, Plumbago zeylanica, Abroma augusta, Abutilon indicum, Sida cordata, Crateva nurvula, Abrus precatorius, Terminalia arjuna, Phyllanthus reticulatus, Azadirachta indica, Tribulus terrestris, Centella asiatica, Strychnos nux-vomica, Alstonia scholaris, Ichnocarpus frutescens, Gymnema sylvestrae, Hemidesmus indicus, Tylophora indica, Solanum surattense, Cuscuta reflexa, andrographis paniculata, Hemigraphis hirta, Eclipta alba, Globba bulbifera, Costus speciosus, Dioscorea pentaphylla, etc. The fern Helminthostachys zeylanica under the family Ophioglossaceae is rare and interesting element observed here.

Table 1. Statistical analysis of Flora of Kulik and its vicinity, Raiganj, Uttar Dinajpur

\begin{tabular}{|cc|c|c|c|c|}
\hline \multirow{2}{*}{ Plant Types } & \multicolumn{4}{|c|}{ Study site is in and around Kulik } \\
\cline { 3 - 6 } & & Class & Families & Genera & Species \\
\cline { 3 - 6 } & & $(1)$ & $(2)$ & $(3)$ & $(4)$ \\
\hline Angiosperm & Dicots & 64 & 184 & 192 \\
\hline Angiosperms & Monocots & 14 & 31 & 32 \\
\hline \multicolumn{2}{|c|}{ Total :. } & 02 & 78 & 215 & 224 \\
\hline Gymnosperms & & - & 6 & 3 & 3 \\
\hline Pteridophytes & Total: & & 85 & 225 & 234 \\
\hline \multicolumn{2}{|c|}{} & \multicolumn{2}{|c|}{} & & \\
\hline
\end{tabular}

Table 2. Statistical Analysis of number of Genera and species in the study area with 5 dominant families

\begin{tabular}{|l|c|c|c|}
\hline \multicolumn{1}{|c|}{ Families } & Genera & Species & Position \\
\hline Asteraceae (Dicots) & 13 & 13 & I \\
\hline Euphorbiaceae (Dicots) & 07 & 09 & II \\
\hline Mimosaceae (Dicots) & 06 & 08 & III \\
\hline Poaceae (Monocots) & 08 & 08 & IV \\
\hline Araceae (Monocots) & 04 & 04 & V \\
\hline TOTAL: 5 & 38 & 42 & \\
\hline
\end{tabular}


Figure 1. Pie diagram showing families, genera and species available in the study area.

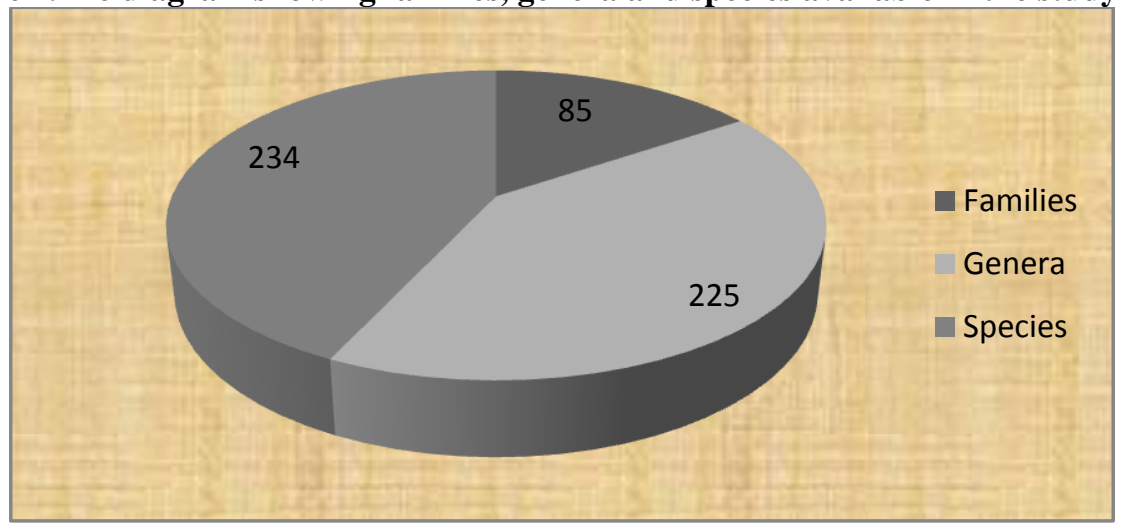

Figure 2. Bar diagram showing 5 dominant families as per the genera and species.

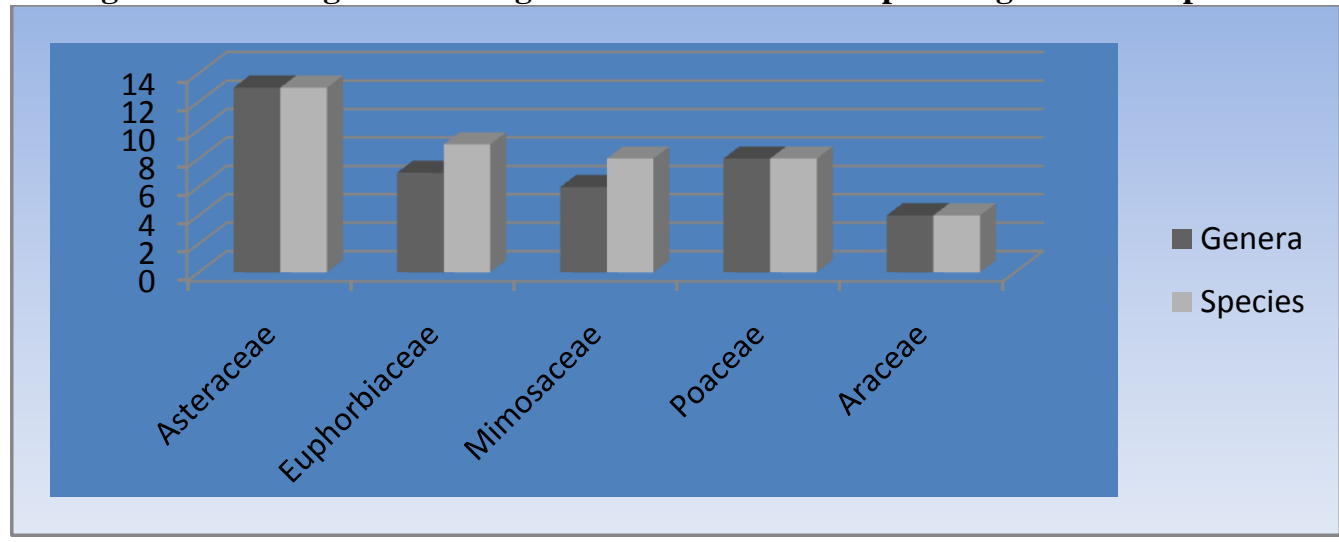

VI. Photo Plates 1-13 (Figures 1-30)
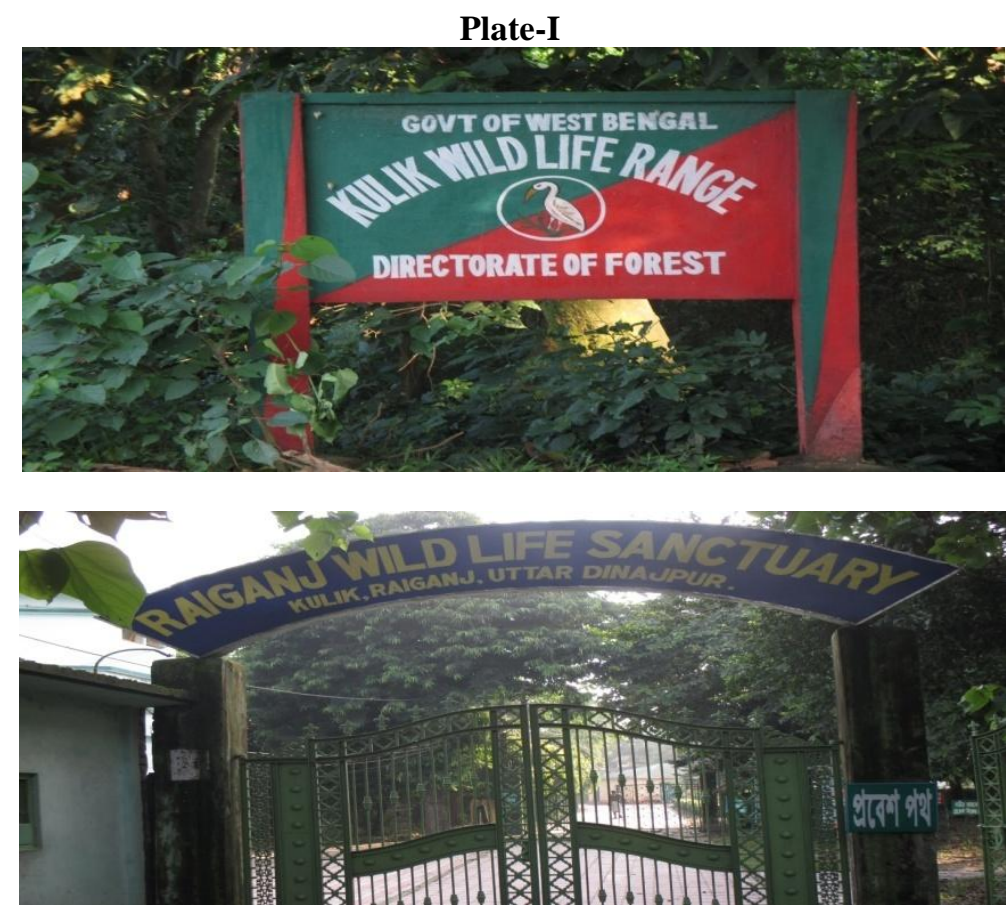


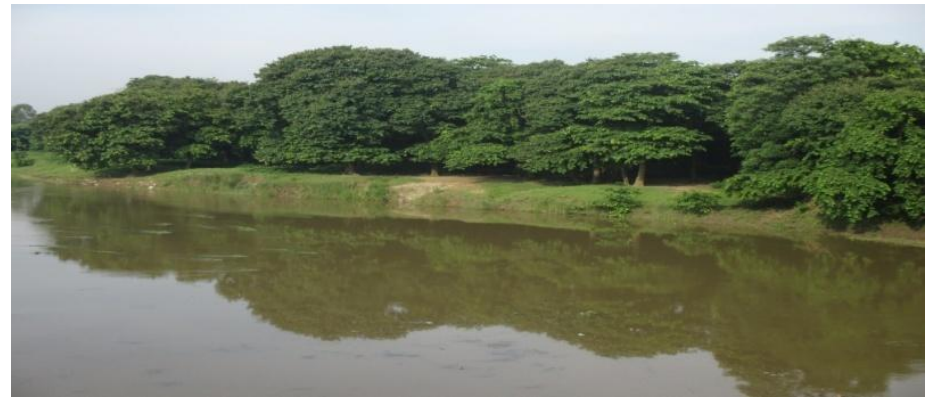

Photo (1-3) : Site marked for Sanctuary, Gate way of Sanctuary, Kulik Sanctuary surrounded by River Kulik from Raiganj towards Siliguro, lefthand side of the road NH-34, at Uttar Dinajnpur District, West Bengal, India

\section{Plate-II}

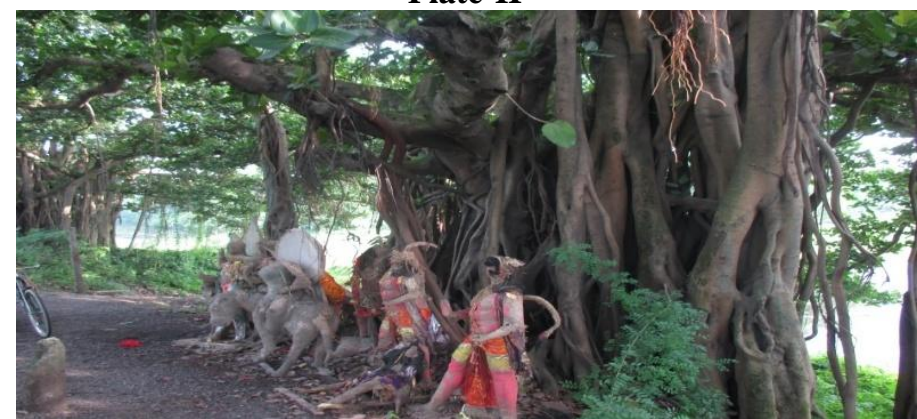

Photo 4: Flagship species Ficus benghalensis (Bengali Name Bat)

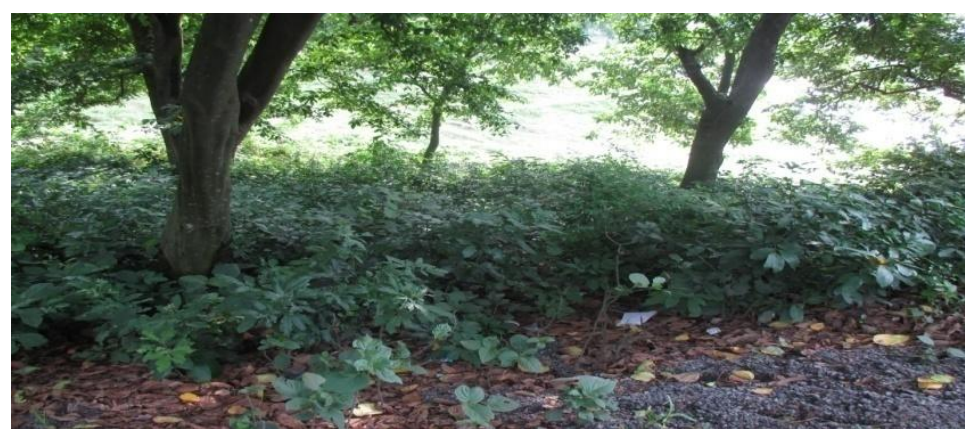

Photo 5.: Underground vegetation dominated by Glycosmis pentaphylla (Bengali Name : Banlebu)

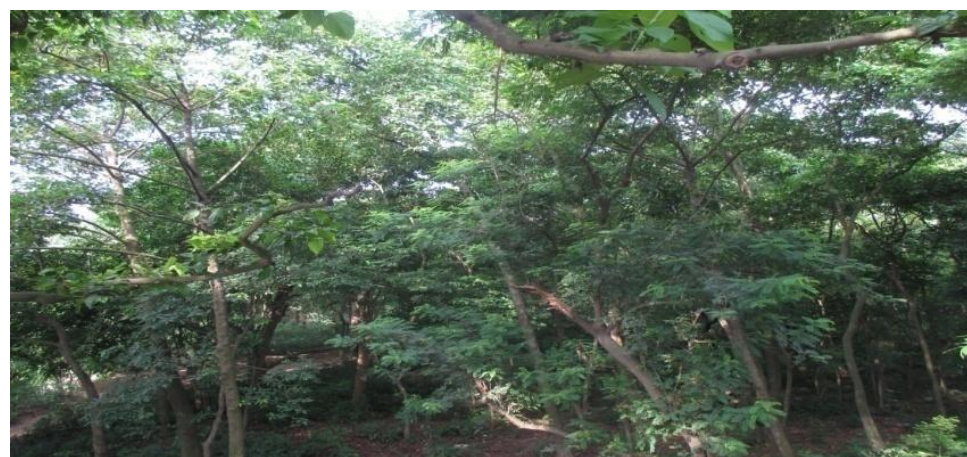

Photo 6. : Tree species like Cassia siamea of Caesalpiniaceae at Social Forestry site, Kulik 


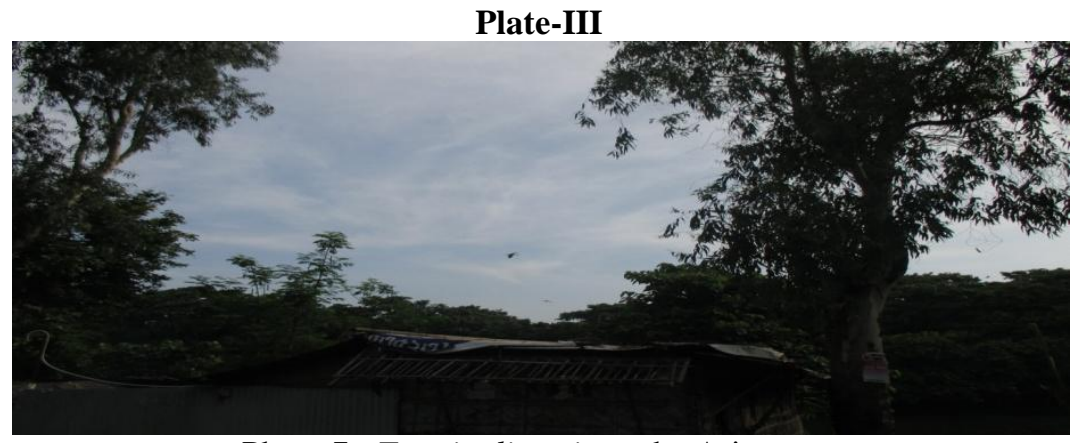

Photo 7.: Terminalia arjuna-the Arjun tree

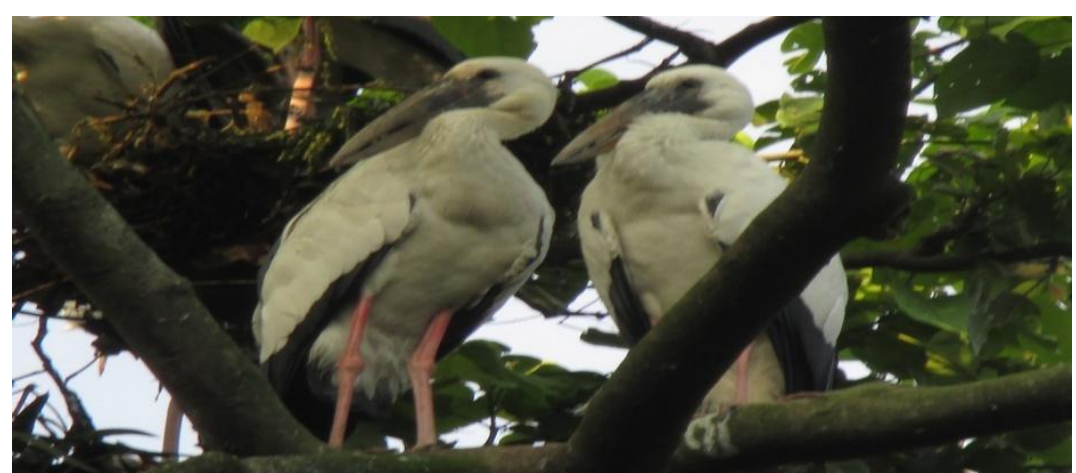

Photo 8.: Open Bill Storks in Resting State on Trewa nodiflora Tree (Family: Euphorbiaceae)

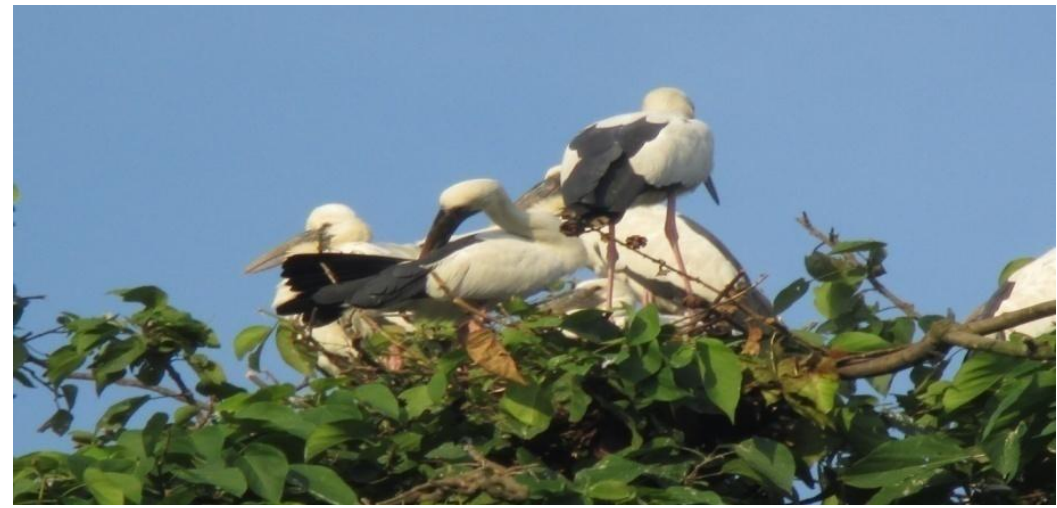

Photo 9.: Open Bill Storks in Resting State on Lagerstroeimia speciosa Tree (Family: Lythraceae)

Plate-IV

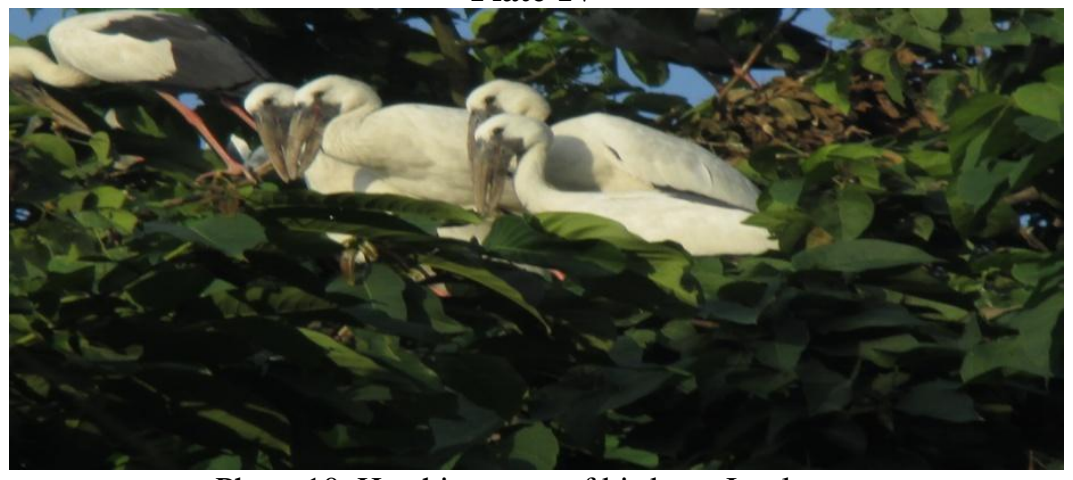

Photo 10. Hatching state of birds on Jarul tree 


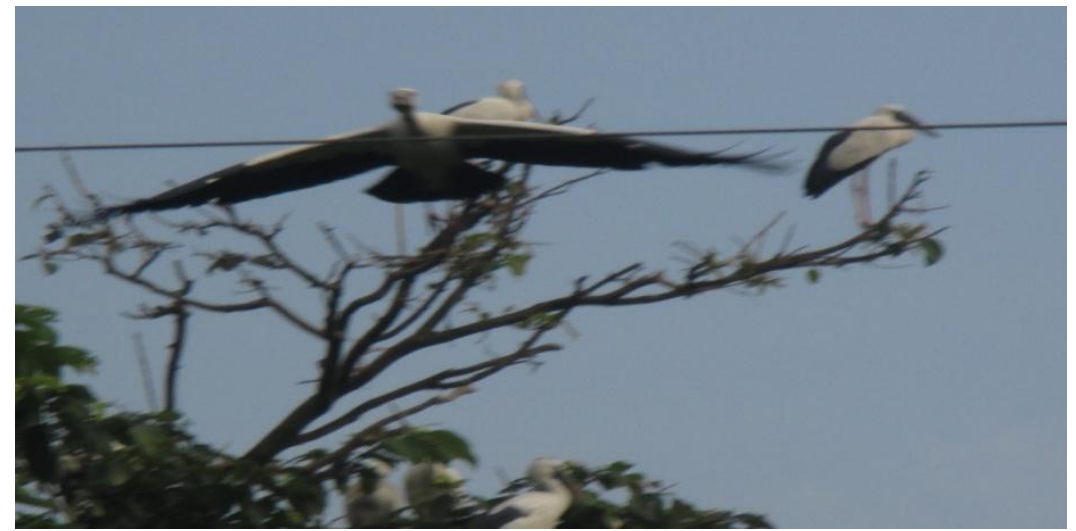

Photo 11. Gliding of Bird

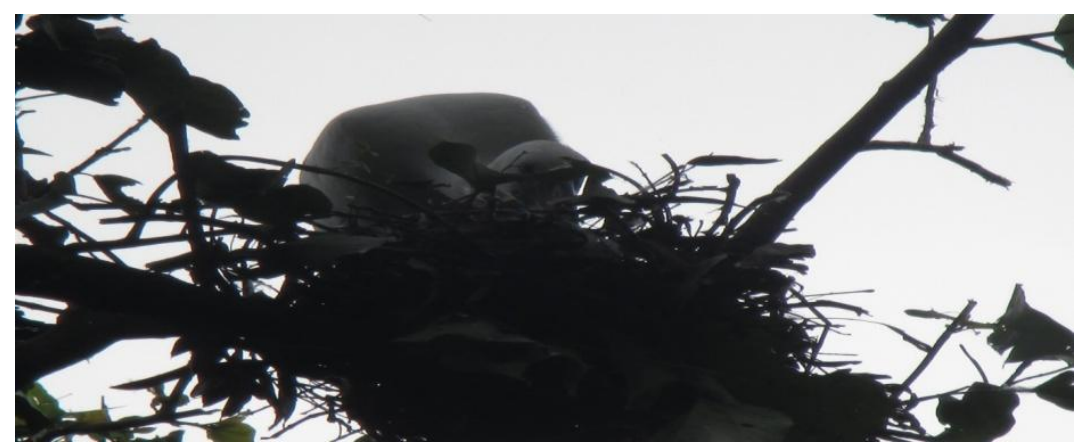

Photo 12. Working in the nest for hatching eggs.
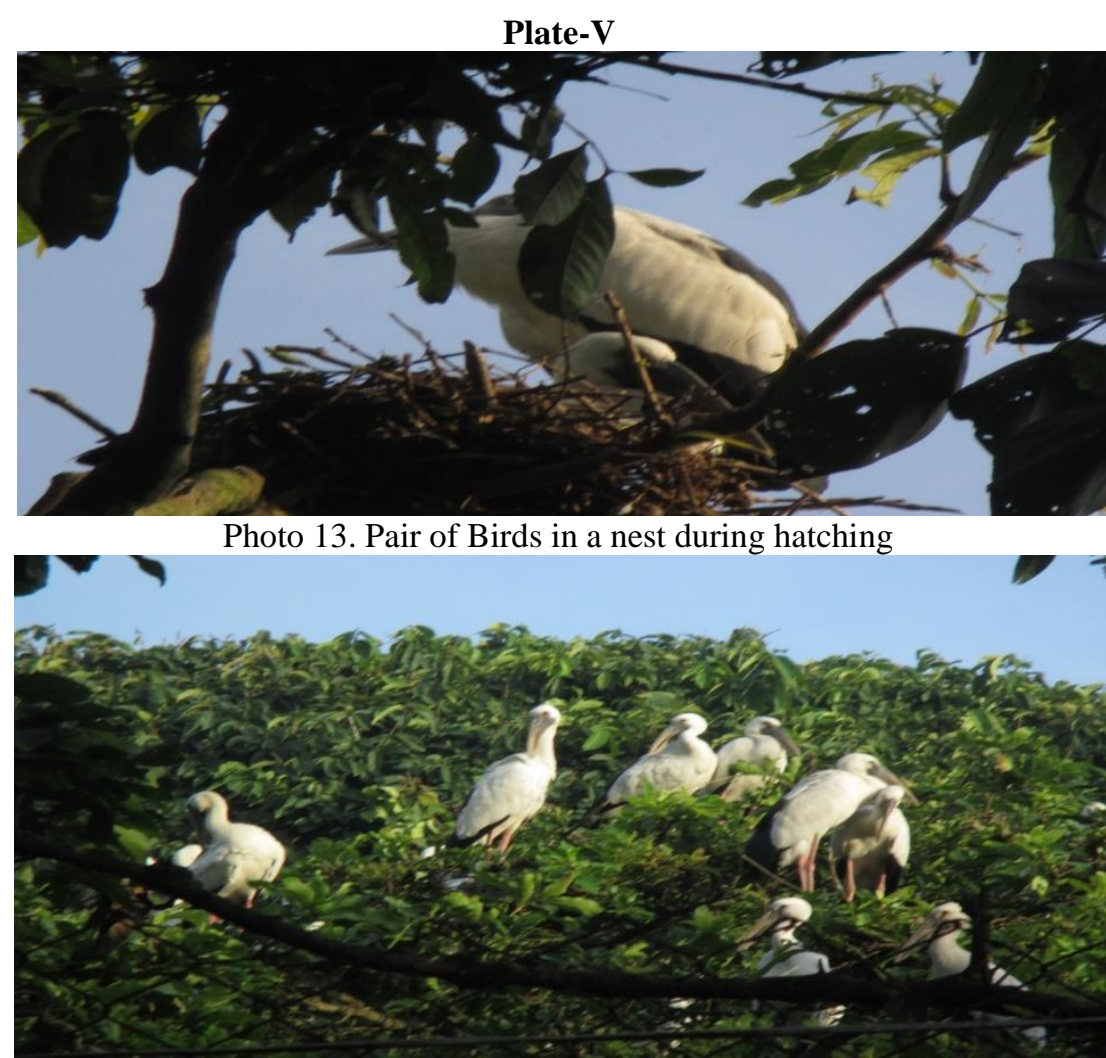

Photo 14: Open Bill Storks in Resting State on Barringtonia acutangula Tree (Family: Lecythidaceae) 


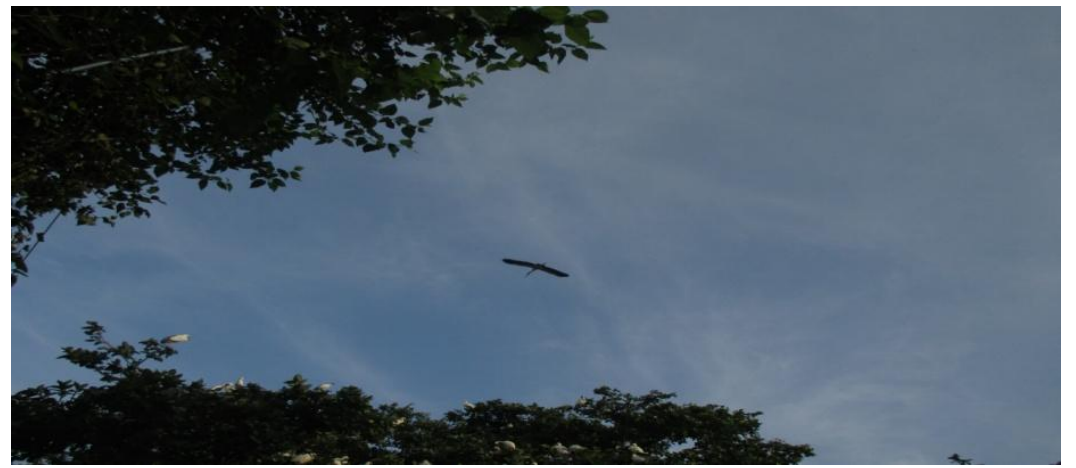

Photo 15: Gliding of Open Bill Stork in the open sky and some are sitted on the Banyan tree (Family: Moraceae) Plate-VI

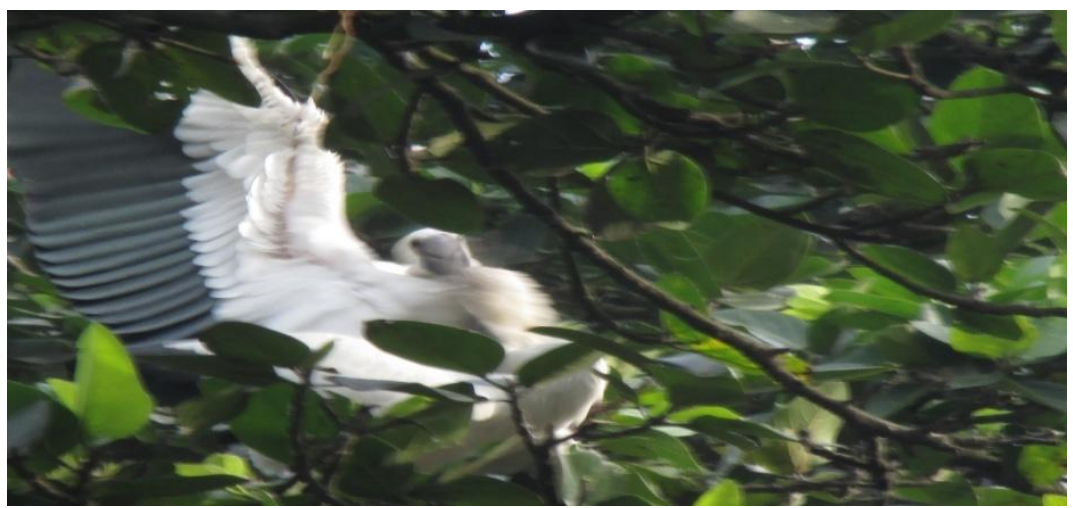

Photo 16: Relasing old feathers for refreshment on Banyan tree

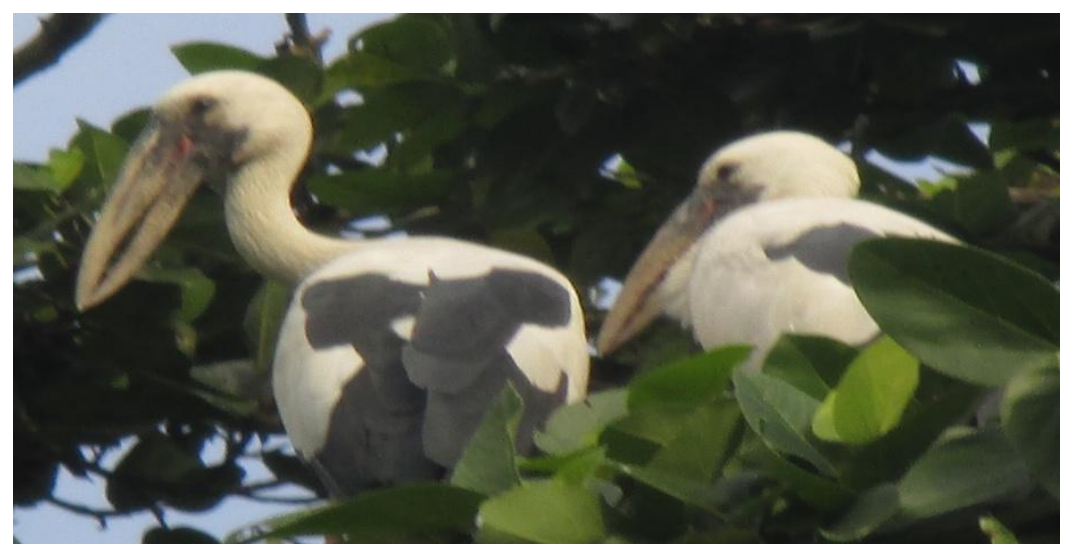

Photo 17: Habit picture of birds at special habitat on tree

\section{Plate-VII}

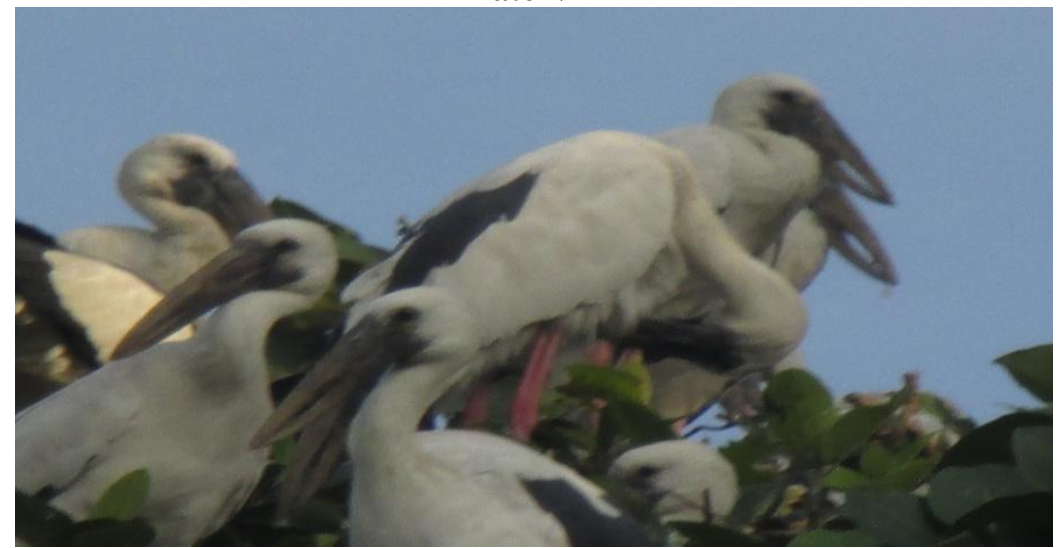

Photo 18: Association of migratory birds on big tree species 


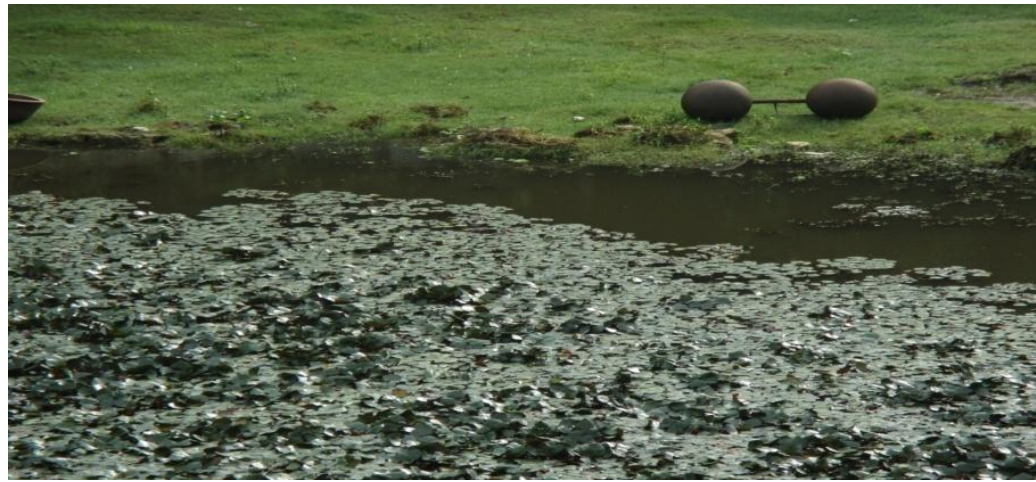

Photo 19: Wetland with Trapa bispinosa of Trapaceae a source of income for local inhabitant.

Plate-VIII

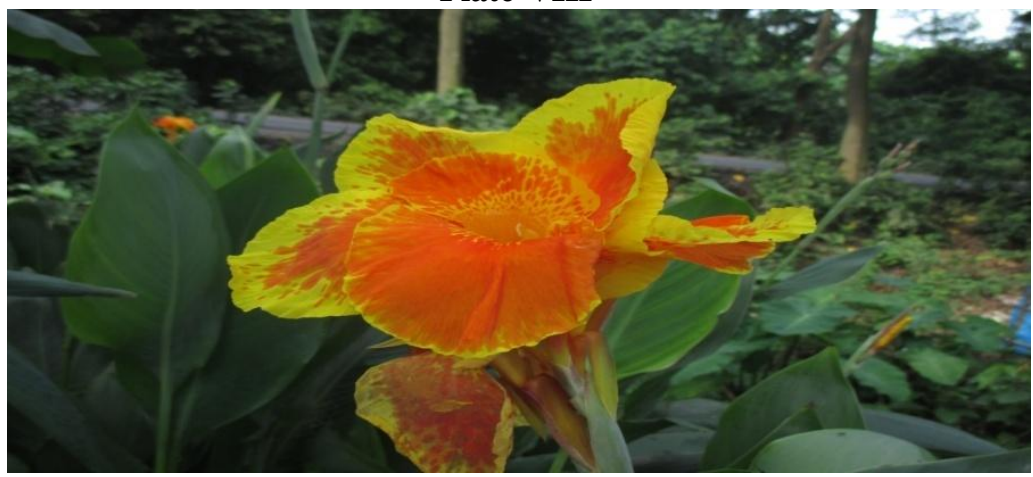

Photo 20: Canna indica of Cannaceae as ornamental one in nearby village

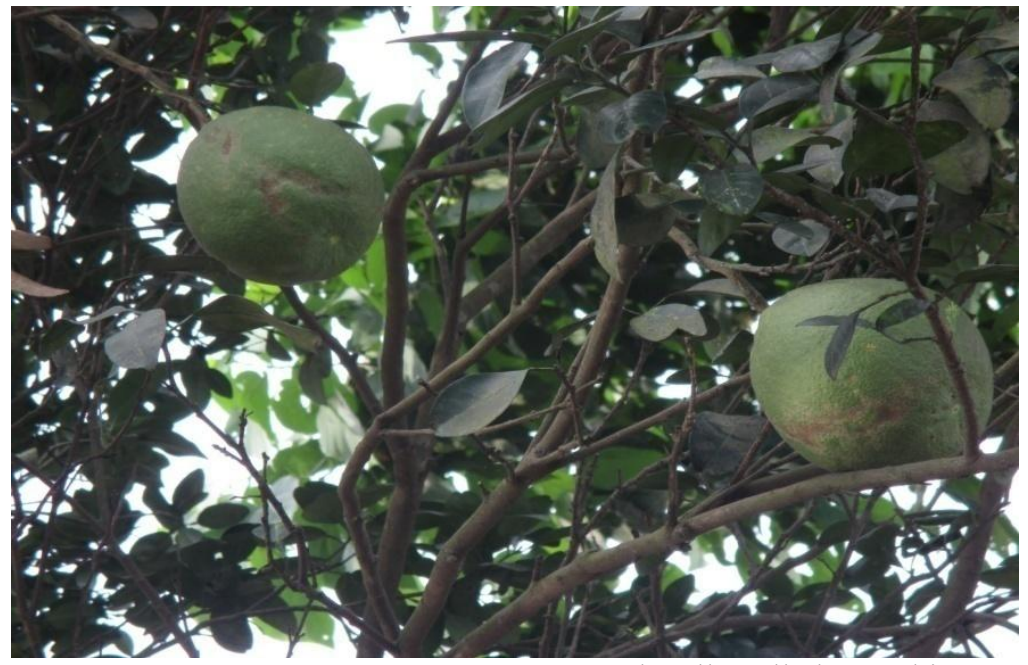

Photo 21: Citrus decumana of Rutaceae-locally called "Batabi"

Plate-IX

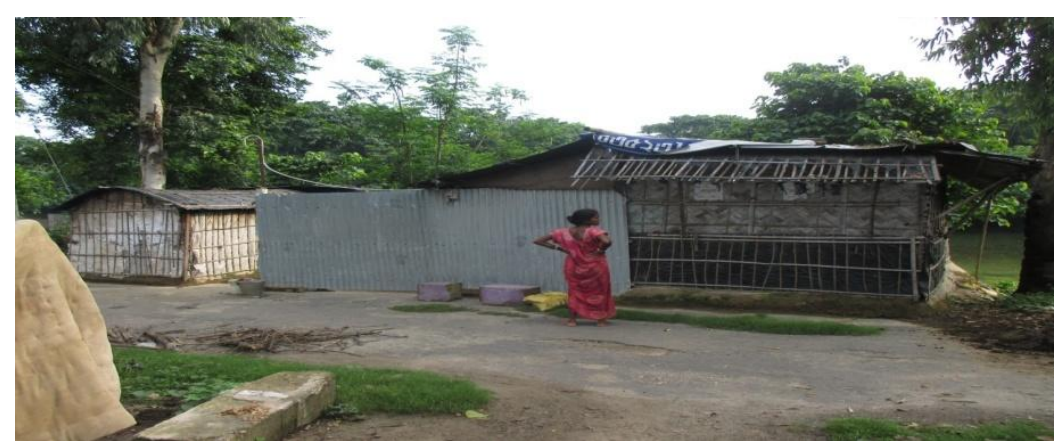

Photo 22: Huts near Kulik 


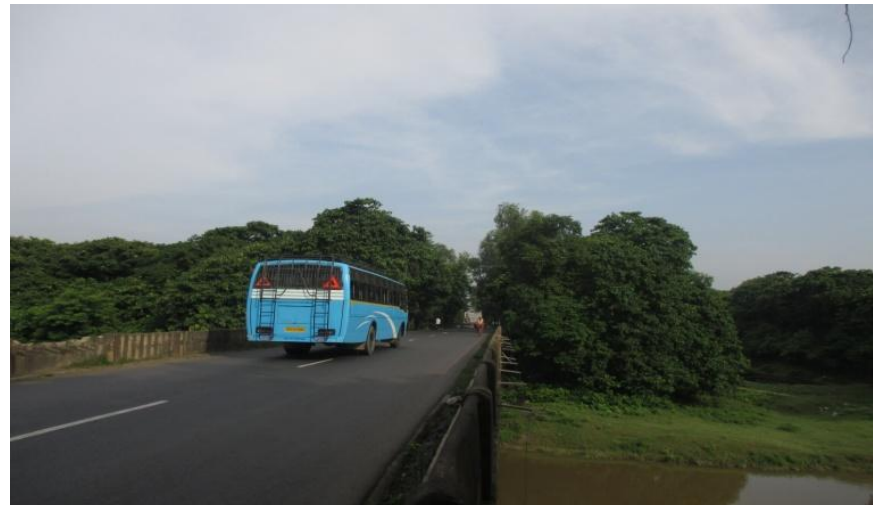

Photo 23: River Kulik , an old bridge a gateway of Kulik from Raiganj, Uttar Dinajpur, West Bengal, India

\section{Plate-X}

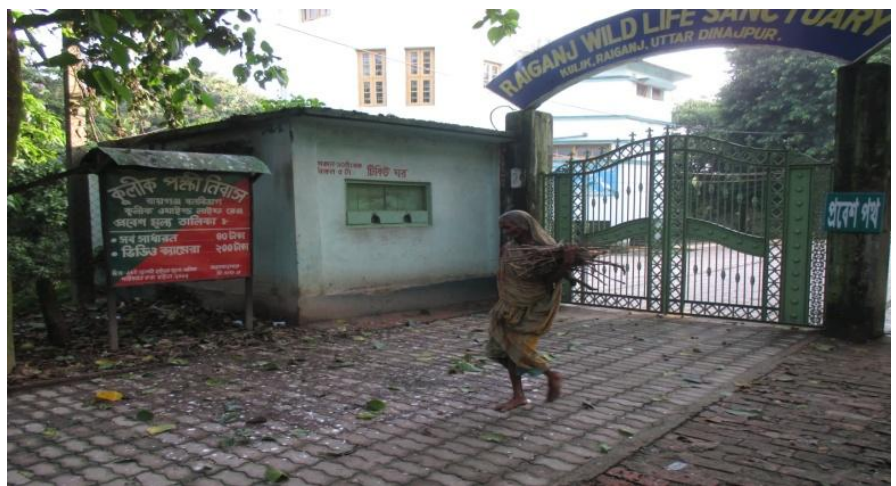

Photo 24: Old women carrying fallen twigs and litter for fuel purpose.

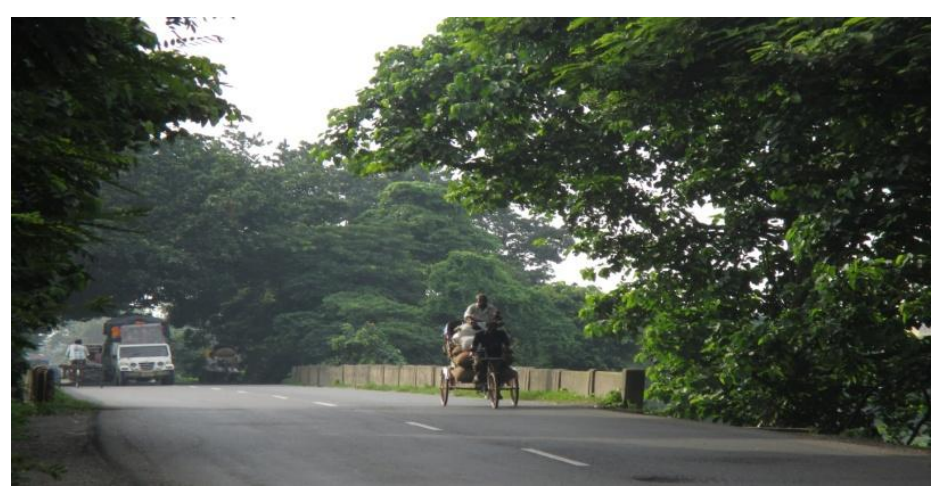

Photo 25: Canopy cover of Vegetation along the NH-34 at Kulik, Raiganj.

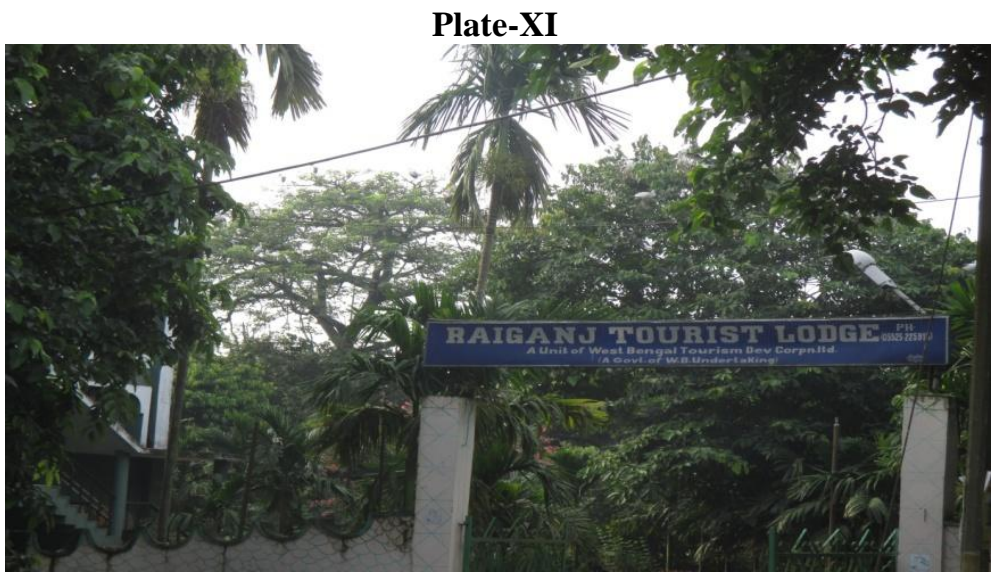

Photo 26: Tourist Lodge at Raiganj, U/Dinajpur a special habitat with big trees 


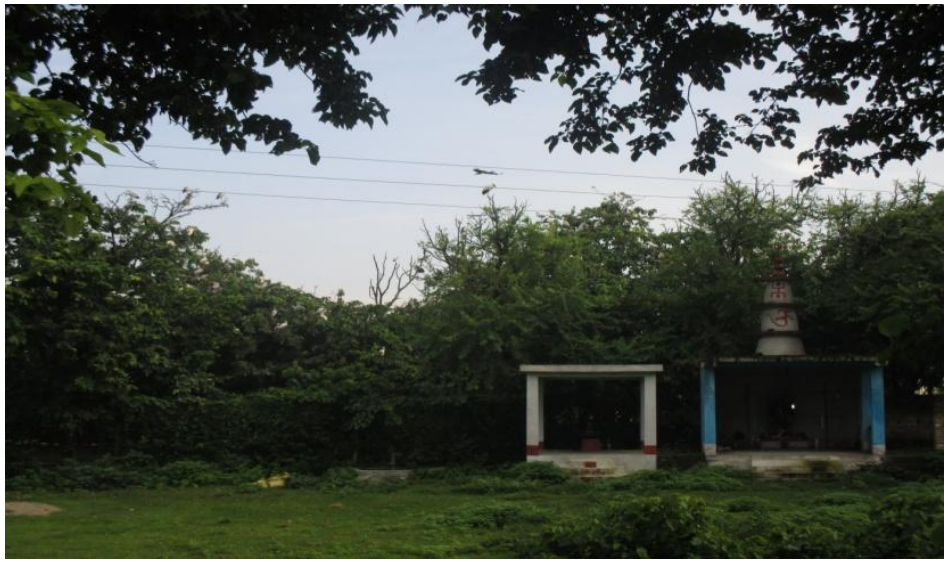

Photo 27: Temple of Lord Shiva (Bengali :Shiv Mandir) at Kulik, Uttar Dinajpur (U/D)

\section{Plate-XII}

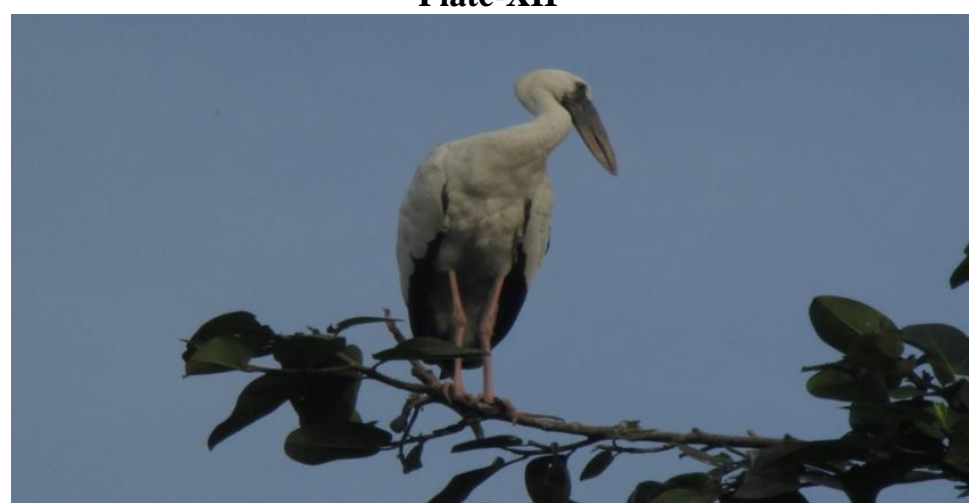

Photo 28: Full picture of an Open Bill Stork (Front face)

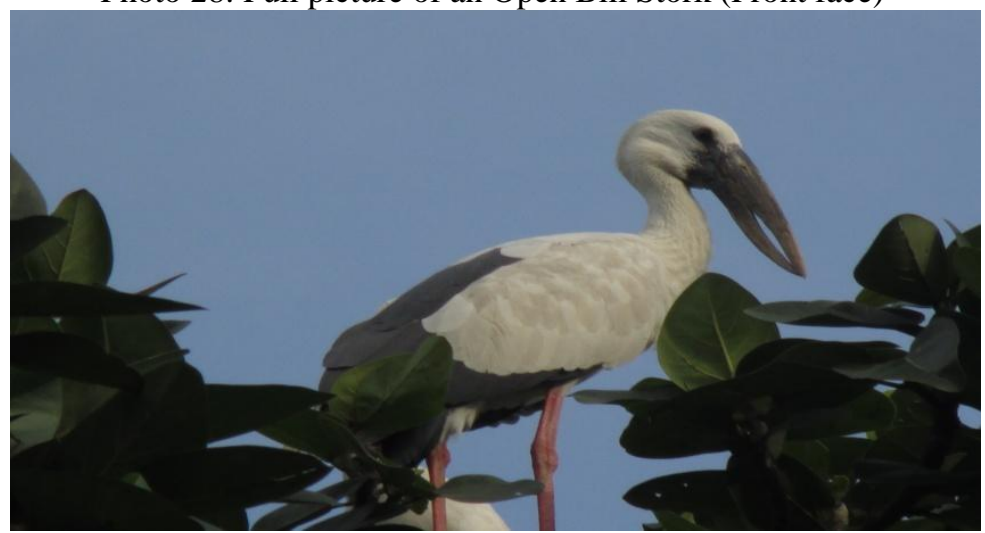

Photo 29: Full picture of an Open Bill Stork (Lateral view)

\section{Plate-XIII}

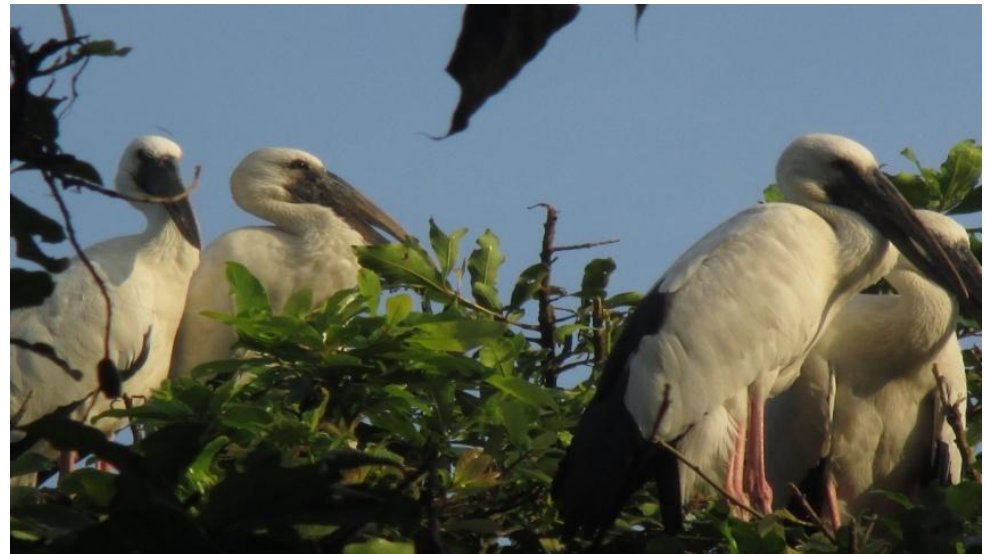

Photo 30: Storks are in Standing and resting position on Hijal tree 


\section{Summary and Conclusion}

In and around Kulik, 5 dominant plant families are Asteraceae, Euphorbiaceae, Mimosaceae, Poaceae and Araceae are well perceived. Among them, co-interaction takes place in between families Asteraceae and Poaceae which are cosmopolitan in distribution. The study is not confined because more crucial efforts should be given to study as a whole, because invasiveness gradually increasing so, study in connection with monitoring is required even to need the study of structure, function and dynamics of vegetation in and around Kulik. This is time consuming even expensive, so funding is required and more critical study through department must be involved. Not only that, there should be one herbarium which will be enriched through thorough study by different workers time to time. At a glance it must be dynamic through the passing of time, so more and more researchers will come to the spot to study more in a common platform to study the diversity of plants, animals as well as culture for the same site.

\section{Legends:}

Graph 1. Pie diagram represents Families, Genera, and species of plants at a glance found in Kulik Bird Sanctuary, Raiganj, U/D.

Graph 2. Bardiagram represents dominat plant families in terms of species composition available at Kulik Bird Sanctuary, Raiganj, U/D.

\section{Acknowledgements}

The authors express their sincere thanks to the people of Sohari and Abdulghata village, Raiganj, U/D for their cordial help during field study. Foresters, experts and social workers are well acknowledged. Head of the P.G. Department of Botany, Darjeeling, some friends of CNH, BSI, Kolkata are well acknowledged. We convey thanks to Sri Parthasarathi Nandi, Zoology Deptt of Raiganj College, U/D for help as and when required. We acknowledged our daughter Atmaja Avirupa Das, a student of Class-I, VSN, who helped us much during field visit including data analysis.

\section{Journal Articles:}

\section{References}

[1]. Basu, P and Sah, R. Census of Temporal variation in the Arrival of Asian Open Bill Stork population: A Case study of Kulik Wildlife Sanctuary, West Bengal, India., Int. Res. J. Environment Sci, 2(4), 2013, 39-43

[2]. Pramanik, A K; Santra, K B and Manna, C B. Nest-Building Behaviour of the Asian Open Billed Stork Anastomus oscitanus, in the Kulik Bird Sanctuary, Raiganj, India, Our Nature, 7(1), 2009, 39-47., DOI: 10.3126/on.v7il2552

[3]. Chakraborty, M R; Chakrabarty, J and Chatterjee, N C. Phytodiversity at Bethuadahari Sanctuary, Nadia, West Bengal, Environment and Ecology, 22 (Spl-3), 2004, 438-441.

[4]. Das, D. Community study of plant species in coastal areas of Mohana and Old Digha of PurbaMedinipur District with special reference to Eco-sustenance of life in near future, Indian J. App. \& Pure Biol., Vol.-29 (2), 2014.

[5]. Ghosh, P. Preliminary studies on Ethno-botanically important Non Timber Forest Produces (NTFPs) in Jamboni Block of Paschim Medinipur District of West Bengal, IOSR-Journal of Pharmacy and Biological Sciences, 9(5), 2014, 59-66.

[6]. Das, D. and M. Das. Vegetation Ecology of Coastal belt of Khejuri area of Purba Medinipur District with special reference to Hijli Coast, West Bengal, India, IOSR-Jour of Pharmacy, 4(2), 2014, 2319-4219.

[7]. Ghosh, P. and D. Das. Some medicinal Plants of Joypore forest Range of Bankura, West Bengal, India, Environment \& Ecology, Kalyani, Nadia, W.B, 32(2), 2014b, 465-470.

\section{Abstracts:}

[8]. Ghosh, P. Biodiversity of Plant Species in and around Kulik: A Case Study $-1^{\text {st }}$ Phase, XI th West Bengal State Science and Technology Congress, 2004.

\section{Books:}

[9]. Anonymous. Ethnobotany and Medicinal Plants of Indian Sub-Continent, Editor-J K Maheswari, Vedams Books for India, Scientific 2000, XI 6728, ISBN: 8172332211 (In the book Papers two by R B Ghosh and Debabrata Das, Nos.: 70, 71).

[10]. Cronquist, An Integrated system of Classification of Flowering Plants, Columbia University Press, New York, USA., 1988.

[11]. Dennis, J.G. and Ruggiero, M.A. Biodiversity Inventory: Building and Inventory at scale from local to global. In: Szaro, R.C. and Johnston, D.W. (Eds.). Biodiversity in Managed Landscapes., Oxford Univ. Press, Oxford, 1996, pp. 149-156.

[12]. Solbrig, O.T.(Ed.). From genes to ecosystems: A Research Agenda for Biodiversity, IUBS, Paris, 1991.

[13]. Prain, D. Bengal Plants, Vol.-I, (Revised Edn, 1903 ), 1963, BSI, Calcutta

[14]. Prain, D. Bengal Plants, Vol.-II, (Revised Edn, 1903), 1963, BSI, Calcutta

[15]. Haines, H. H. The Botany of Bihar and Orissa, Vol. I-IV, (1921-1925), BSI, Calcutta.

[16]. Hooker, J. D. Flora of British India, Vol. 1-VII, (1892-1897), BSI, Calcutta.

[17]. Anonymous. Medicinal Plant Resources of South West Bengal, Vol.-I, 2005, Research Wing, Directorate of Forests, Govt. of West Bengal.

[18]. Anonymous. Medicinal Plant Resources of South West Bengal, Vol.-II, 2010, Research Wing, Directorate of Forests, Govt. of West Bengal.

[19]. Anonymous. Flora of West Bengal, Vol.-I , BSI, Kolkata, Flora of India, 1997, Series-2

[20]. Groom, M. J; Meffe, G. K ; Carroll, C. R. and Contributors. Principles of Conservation Biology, Third Edition, Sinauer Associates, Inc. Publishers, USA.,2006, pp.-793.

[21]. Rao, R. R. and Sharma, B. D. A Manual for Herbarium Collections, BSI, Brabourne Road, Kolkata-1., 1990.

[22]. Mabberley, D. J. A Portable dictionary of the Vascular Plants , Cambridge University Press, 1997.

[23]. Bennet, S. S. R. Name Changes in flowering plants of India and adjacent regions, Triseas Publishers, Dehra Dun, India, 1987. 
Thesis:

[24]. Das, D. Study of Vegetation Ecology of Forests of lateritic Southwest Bengal with special reference to Non-Timber Forest Produce (NTFP) Productivity, Ph. D Thesis awarded from Vidyasagar University, West Bengal (Work From CNH, Botanical Survey of India, Shibpore, Howrah, West Bengal), 2007.

Other Articles:

[25]. Anonymous. Leaf let report of the forest department,2004, Karnajora, Uttar Dinajpur, West Bengal.2004.

[26]. Sharma, A. The Asian Open-Bill Stork Thrives in the Kulik Bird. The Statesman, Daily India, August publication, 2007.

[27]. Raiganj-Wikipedia, the free Encyclopedia:en.wikipedia.org/wiki/raiganj

[28]. Anonymous. Uttardinajpur.hpage.co.in/information-6368829.html.

[29]. Stohlgren, T.J. and Quin, J.F. Status of National Resources Data Bases in National Parks: Western Region., National Park Service, Co-Operative Park Studies Unit, Technical Report 44., University California, Davis, CA, 1991. 